\title{
Más mujeres en las ciencias: estrategias para aumentar su participación
}

More women in science: strategies to increase their participation

\section{MARÍA CLAUDIA SEGOVIA-SALCEDO 1,2 [D}

Universidad de las Fuerzas Armadas ESPE, Departamento de Ciencias de la Vida y la Agricultura.

Av. Rumiñahui s/n y Calle Ambato. Correo electrónico mcsegovia@espe.edu.ec

2 Red Ecuatoriana de Mujeres Científicas REMCI

\section{RESUMEN}

La investigación se centra en la generación de conocimiento. Sin embargo, por mucho tiempo esta generación se ha enfocado en dar respuestas a problemas y preguntas de solo una porción de la población debida, en parte, a una falta de presencia femenina. Y aunque la participación de las mujeres y las niñas en las ciencias ha mejorado en nuestro país y a nivel mundial, ciertas áreas de las ciencias siguen siendo dominadas por hombres y no hemos alcanzado la equidad. En este trabajo analizamos tres estrategias para incrementar la presencia de las niñas y las mujeres bajo la perspectiva de nuestro país: generar un ambiente de trabajo inclusivo, eliminar la discriminación y reducir la brecha de pago.
Palabras claves: Mujeres en ciencias, sesgo de género, estrategias, inequidad

\section{ABSTRACT}

This Research focuses on knowledge generation. However, since a long time, this generation has focused on providing answers to problems and questions, which belong to a portion of the population only, due in part, to a lack of female presence. And although women's and girls' participation in science has been 
improved in our country and all over the world, certain areas of science continue to be subjected by men and we, women, have not achieved equity in gender. In this paper, we analyze three strategies to increase women's and girls' presence under the perspective of our country: to create an inclusive working environment, to eliminate discrimination, and to reduce the payment gap.

Keywords: Women in science, gender bias, strategies, inequity

\section{PROBLEMA Y TENDENCIAS}

La investigación se centra en la generación de conocimiento porque desarrolla conceptos, teorías y modelos dentro de proyectos de tecnología y desarrollo (OECD, 2015). No obstante, por mucho tiempo la generación de conocimiento se ha centrado en dar respuesta a problemas y preguntas de solo el cincuenta por ciento de la población mundial. Y aunque la participación de las mujeres y las niñas en las ciencias ha mejorado en las últimas décadas, las áreas de la ciencia, la tecnología, la ingeniería y la matemática siguen siendo dominadas por hombres (Unesco Institute for Statistics, 2019). Varias estrategias han permitido que más mujeres lleguemos a trabajar en investigación, pero todavía seguimos un paso atrás al momento de la obtención de becas, fondos de investigación y patentes comparadas a nuestros colegas (De Kleijn, y otros, 2020) ¿̇Qué pasa en el Ecuador en relación a este tema? ¿̇Por qué no tenemos más mujeres dedicadas a la investigación y a la academia? ¿Qué podemos hacer para aumentar el número de niñas y mujeres en las ciencias?
Los datos de los últimos años demuestran que la tasa de matriculación en la educación superior del Ecuador ha aumentado en $0.6 \%$ entre el 2014 al 2015, y se ha mantenido esta cifra hasta ahora (Secretaria Técnica Planifica Ecuador, 2019). Igual en el caso de los recursos humanos relacionados a ciencia y tecnología, que han aumentado en la última década en un $40 \%$ a nivel de Iberoamérica, pero seguimos manteniendo las tendencias de un reducido número de maestrías (13\%) y doctorados (1\%) del total de graduados de la educación superior (Organización de Estados Iberoamericanos. Observatorio CTS, 2020). Se demuestra así que el patrón mundial de una disminución de la presencia de las mujeres en los niveles avanzados de las carreras académicas sigue presente también en nuestro país. Encuestas a nivel mundial demuestran que si bien las mujeres no están formalmente excluidas de las oportunidades de educación en los diferentes niveles, estereotipos sociales y conceptos tradicionales sobre el rol dentro de la sociedad generan una exclusión informal de las niñas y mujeres (Dimitriadi, 2013).

En el Ecuador, el número de investigadores se ha incrementado siguiendo las tendencias internacionales, pero todavía se mantiene en niveles bajos comparado a otros países de Latinoamérica (Zambrano Mendoza, 2019). Apenas 2556 investigadores se encuentran acreditados en la SENESCYT hasta el 2018, que corresponde a un 0.30 de investigadores por cada 1000 habitantes, superando únicamente a Bolivia (0.29) y Perú (0.20), en Sudamérica. El 35\% es mujer, porcentaje inferior a los promedios de América Latina y Caribe (44\%) e Iberoamérica (47\%) (Organización de Estados Iberoamericanos. Observatorio CTS, 2020; Zambrano Mendoza, 2019; UNESCO, 2015). Y 
en el Ecuador, en el área académica donde agrupa a la mayor cantidad de investigadores, solamente 3 de cada 10 docentes con Ph.D. son mujeres (Herdoíza, 2015).

\section{DISCRIMINACIÓN}

La participación de la mujer en el ámbito laboral, y en trabajos relacionados de ciencia y tecnología ha aumentado en los últimos años en Ecuador e Iberoamérica. Las mujeres representan el $42 \%$ del total de docentes en Iberoamérica en 2018, datos que difieren de la participación femenina como estudiantes y graduadas que son mucho más altos (Organización de Estados Iberoamericanos. Observatorio CTS, 2020). Y a pesar de que los datos nos demuestran un incremento en la presencia femenina en diferentes áreas, se siguen registrando tendencias bajas en las ciencias e ingenierías. Incluso en el caso de las finanzas y empresas donde apenas un 30\% de los puestos de decisión es ocupado por mujeres (Espinosa, 2019).

Sabemos que la ciencia está fuertemente enfocada al género masculino, lo que lleva a una distribución inequitativa de recursos entre hombres y mujeres en las diferentes áreas de investigación (Nosek, Banaii, \& Greenwald, 2002). Esto responde, también, a un contexto social y cultural donde los estereotipos de roles de género orientan a las niñas a ser más comunitarias, enfocadas en los amigos y la familia en actividades donde se fortalecen las relaciones interpersonales. Mientras, en el caso de los niños, los orientan a ser más atléticos, a explorar la parte física, entender cómo funcionan las cosas, y poner énfasis en la resolución de problemas, el estatus y las ganancias (Dimitriadi, 2013; Weisgram, 2019). Por lo que, las carreras relacionadas a matemáticas y ciencias son percibidas como masculinas (Makarova, Aeschlimann y Herzog, 2019). Adicionalmente, en nuestro contexto, la carrera de investigación es poco reconocida y atractiva, como lo demuestran los datos de Zambrano Mendoza (2020).

En el Ecuador, el número de investigadores ha crecido a un ritmo mucho más lento que otras profesiones en la última década. La falta de motivación e incentivos frente a la ciencia y tecnología han llevado, incluso, a que el número de investigadores no esté relacionado con el número de publicaciones, como sucede en la mayoría de los países de Latinoamérica, donde a mayor número de investigadores, tenemos más publicaciones (Zambrano Mendoza, 2020). En el caso de las mujeres, debemos añadir la discriminación, muchas veces inconsciente, como una de las razones de las bajas cifras. Esta discriminación nace de los roles y estereotipos culturales que llevan a que las mujeres sean las principales responsables de las tareas del hogar, la crianza de los hijos y el cuidado de los adultos mayores, especialmente dentro de los países de Latinoamérica.

Esto conlleva a una reducción en el tiempo dedicado a su carrera y profesión, la poca participación en la toma de decisiones y puestos de decisión, y que las mujeres ocupen empleos reconocidos por la sociedad como inferiores (Secretaria Técnica Planifica Ecuador, 2019). Otros estudios sugieren que los estereotipos de género en las áreas de ciencias y tecnologías pueden influir de manera temprana en las aspiraciones de las chicas al momento de decidir sus carreras (Makarova, Aeschlimann, \& Herzog, 2019). Investigaciones demuestran que todos tenemos sesgos, incluso al momento de invitar 
a expositores a charlas académicas, lo que implica un menor acceso a redes, proyectos, colaboraciones en publicaciones y también en potenciales ofertas de trabajo (Nittrour, y otros, 2018).

A nivel global, los hombres dominan la producción científica con un $70 \%$ frente a un $30 \%$ de las mujeres. Además, los artículos publicados por mujeres como primera o como única autora reciben menos citas, si los comparamos con autores en las mismas posiciones (Lariviere, Chaoquin, Yves Gingras, \& Sugimoto, 2013). Incluso, los hombres manejan sus citas de diferente manera que las mujeres, autocitando sus trabajos en mayor cantidad que las mujeres (56\%) aumentando los índices de citación, ampliando las brechas en las citas y generando un ventaja acumulativa (King, Bergstrom, Correll, Jacquet, \& West, 2017). También se detectan sesgos en las cartas de recomendación, donde las mejores cartas las reciben los postulantes masculinos (Dutt, Pfaff, Bernstein, Dillard, \& Block, 2016) y en los procesos de contratación, a pesar de tener comisiones equitativas y las mismas calificaciones de los candidatos, se da la preferencia a los postulantes masculinos. Igual en el Ecuador: lo demostraron en un diagnóstico en una universidad ecuatoriana, que a pesar de tener un $62 \%$ del estudiantado femenino, a 2013 , solo el $20 \%$ del cuerpo docente era mujer (Herdoíza, 2015).

También las mujeres deben enfrentar un ambiente violento no solo en sus hogares sino en sus lugares de trabajo. Según el informe de PNUD-ONU Mujeres (2017), América Latina es la región más violenta del mundo para las mujeres. Ecuador ocupa el tercer lugar en Sudamérica en estas tristes estadísticas. Datos de 2011 visibilizaron que un 55\% de las mujeres ecuatorianas mayores a los 15 años ha experimentado algún tipo de violencia (Secretaria Técnica Planifica Ecuador, 2019). La naturalización de las agresiones ha producido que la violencia no sea percibida en diferentes ámbitos, y la ciencia y academia no son excepciones, por lo que el porcentaje de denuncias es bajo (Altamirano, 2020; De Kleiin, y otros, 2020). En el caso de estudiantes, Ibañez (2017) encontró que un $56 \%$ de las afectadas por acoso no denunció y muchas de ellas abandonaron sus estudios.

En este ensayo se pretende explorar diferentes estrategias para mejorar la situación de las mujeres en la academia y en las ciencias. Estas estrategias se enfocan en alternativas para cambiar el ambiente no siempre inclusivo para las mujeres, que ya se ha propuesto en otras partes del mundo y que también se pueden aplicar en nuestras instituciones.

\section{TRES ASPECTOS}

Cada uno de nosotros podemos hacer cambios en esta realidad y aportar a la mayor presencia de niñas y mujeres en ciencias, y así lograr una ciencia más diversa e inclusiva. En este caso presentamos tres estrategias basadas en el trabajo de Somerville \& Gruber (2020), aplicados a nuestra realidad:

\section{Generar un ambiente de inclusión para las niñas y mujeres}

Las mujeres en las diferentes áreas de ciencia y tecnología no se sienten incluidas en la academia, no se sienten parte del grupo. $Y$ eso se ve reflejado en la falta de respeto y autoridad por parte de sus colegas, así como un menosprecio del trabajo académico 
en las mujeres. Estas experiencias comunes para las mujeres en ciencias pueden conllevar a mayores tasas de deserción a todo nivel (Somerville \& Gruber, 2020; Herdoiza, 2015). A pesar de que estudios demuestran que una diversidad de género produce nuevos descubrimientos por el aumento en la percepción social y de diferentes perspectivas (Nielsen, y otros, 2017).

Una alternativa es visibilizar a las mujeres en ciencias, sus logros y su presencia en cada una de nuestras instituciones y entorno. Es importante que las niñas, estudiantes y jóvenes vean otras mujeres como expositoras, mentoras, lideresas en su área de estudio. Investigaciones demuestran que las mujeres se sienten más incluidas cuando hay mujeres en posiciones de decisión (Dimitriadi, 2013). Esto también se puede aplicar en la representatividad femenina entre los expositores, en los conversatorios, en los medios de comunicación, en los congresos que organizamos. La visibilidad y la mentoría son aportes fundamentales no solo para las jóvenes sino para las científicas que inician su carrera.

Otro aspecto que se puede considerar es cambiar la imagen de las disciplinas, porque muchas áreas de las ciencias están asociadas a lo masculino. Cheryan et al., (2013) demostró en su estudio que un cambio de imagen en las Ciencias de la Computación llevó a un incremento en la población estudiantil femenina. Sin embargo, se debe analizar una intervención más temprana con una mayor inclusión de imágenes femeninas en los libros de texto, en películas, radio, prensa y televisión, porque las percepciones de género son internalizadas desde edades tempranas (Makarova, Aeschlimann y Herzog, 2019). A esto se debe añadir el apoyo y motivación de los docentes, así como la familia y su entorno que juegan un rol primordial para seguir y mantenerse en una carrera científica (Makarova, Aeschlimann y Herzog, 2019)
En el caso de los docentes, desde prekinder hasta la universidad, estar alertas, conscientes y sensibles a estos sesgos de género, es un primer paso para una reforma en el currículo y un cambio institucional. De igual manera, la nueva actitud de los tutores y consejeros estudiantiles que guían a los jóvenes en sus opciones educativas y profesionales. Muchos consejeros no están al tanto de los estereotipos tan marcados que enfrentan las niñas y jóvenes y cómo enfrentarlos. Una de las estrategias, en estos casos, es presentar y promover modelos femeninos locales exitosos que puedan actuar como mentoras en las niñas y jóvenes interesadas en las ciencias (Dimitriadi, 2013).

En el rol de padres, es necesario incentivar y motivar a sus hijos e hijas en las áreas de ciencias y tecnologías. Estudios demuestran que la confianza de los padres en las habilidades matemáticas de sus hijos e hijas, incrementan la confianza de los jóvenes. Y en la medida de lo posible, apoyarles para que participen en actividades extracurriculares relacionadas a ciencias y tecnologías (Dasgupta \& Stout, 2014).

Otra estrategia es la creación y el fortalecimiento de colaboraciones con Museos de Ciencia, Historia Natural, así como Universidades y Escuelas Politécnicas, para que los estudiantes de las escuelas y colegios tengan la oportunidad de ampliar sus opciones profesionales y al mismo tiempo puedan ver las aplicaciones de las carreras en ciencia y tecnología. Otra destreza es la de crear ambientes de aprendizaje no formal como actividades extracurriculares o campamentos de ciencia, donde se enfatice la creatividad, actividades prácticas sin la presión de una calificación (Dasgupta \& Stout, 2014). 
En el ámbito institucional se debe contemplar políticas de inclusión de una cultura de trabajo abierta que motive a sus empleados a expresar su identidad cultural y de género, comentar sus preocupaciones, sin sentirse intimidado (Nielsen, y otros, 2017; Else, 2018). Otra estrategia es mantener análisis continuos del ambiente laboral y estar conscientes que la contratación de un mayor número de mujeres no reduce por sí misma la discriminación y sesgo, y que las mujeres no son las que deben cambiar sino la institución. Por lo que esto necesita estar acompañado con políticas y liderazgos que permitan la diversidad para generar innovación y tecnología (Nielsen, y otros, 2017; Dimitriadi, 2013).

También se sugiere motivar el trabajo colaborativo y la equidad en los grupos. Estudios demuestran que las mujeres no participan comúnmente en trabajos colaborativos, ni lideran estos proyectos y sus publicaciones. Y mucho más si en el grupo de trabajo son minoría. Otras acciones: Promover la generación de redes, la participación en conferencias profesionales. Impulsar la presencia de mujeres en los coloquios y congresos académicos dentro de nuestra institución (Lariviere, Chaoquin, Yves Gingras, \& Sugimoto, 2013; Dasgupta \& Stout, 2014)

\section{Enfrentar el acoso y el bullying}

En el día a día, las mujeres encontramos frecuentemente un comportamiento poco profesional de ciertas personas, que incluye humillaciones, intimidación y acoso e incluso de tipo sexual, que genera un ambiente negativo dentro de la academia colocan a la mujer en posiciones de inferioridad y desigualdad, que no siempre son visualizadas, tratadas y solucionadas por las instituciones (Guarderas et al., 2018).

El bullying puede también ser mucho más sutil, al diseminar rumores maliciosos en relación a la capacidad en el trabajo o frente a ciertas opiniones personales. Muchos supervisores se convierten en "bullies" al designar tareas imposibles de lograr, cargas terribles de trabajo, fechas de entrega inalcanzables o un constante cambios de tareas, lo que hace que muchas veces este tipo de maltrato es muy difícil detectarlo (Else, 2018). En muchos casos, estos escenarios laborales no permiten el crecimiento profesional principalmente de las mujeres. Este tipo de comportamientos es mucho más común de lo que creemos en la academia.

Un estudio enfocado en las universidades del país habla de un $56 \%$ de violencia entre estudiantes y docentes; las áreas de ciencias, matemáticas y estadísticas son las más afectadas. Por otro lado, una investigación centrada en 1264 estudiantes de una universidad ecuatoriana, encontró que cerca de un $40 \%$ de las mujeres sufrió acoso dentro de las instituciones de educación superior (Altamirano, 2020). Si bien la Ley Orgánica de Educación Superior en el Ecuador (LOES) contempla responsabilidades para la institución en este ámbito, y que todas las Universidades cuenten con protocolos para la atención y prevención de la violencia de género, los casos de acoso son tratados internamente y muchos de ellos quedan en la impunidad (Guarderas et al., 2018; Altamirano, 2020).

Ormaza (2013) encontró en su estudio que el acoso y bullying se centran en bromas de contexto sexual donde principalmente las víctimas son mujeres (68.4\%), y estas bromas vienen usualmente 
por parte del docente. Lo que demuestra que en la academia ecuatoriana se mantienen modelos sexistas y androcéntricos conectados con relaciones de poder.

Para eliminar el acoso debemos tomar pasos concretos a fin de desarrollar intervenciones más efectivas. Las instituciones deben tener un mensaje claro y explícito de cero tolerancia al bullying, acoso sexual, y al mismo tiempo asegurar procesos de acompañamiento para las víctimas (Somerville \& Gruber, 2020). Estos procedimientos deben ser aplicados independientemente de la posición del acosador, porque el acoso se da porque la institución lo permite (Else, 2018). De igual manera, todo el personal debe ser entrenado y educado para conocer cómo manejar este tipo de situaciones. Y motivar a que el personal involucrado en investigación científica tenga entrenamiento sobre recursos humanos. Promover y capacitar al personal de las instituciones sobre los derechos de género para generar un proceso de sensibilización (Herdoíza, 2015)

\section{Reducir la brecha de pago entre hombres y mujeres}

A nivel mundial, las mujeres reciben una menor remuneración que sus colegas, y si bien la equidad en el salario se ha incrementado, el proceso ha sido lento en las diferentes áreas, en los últimos 20 años (Nittrour, y otros, 2018) Las razones son mucho más complejas y profundas de lo que imaginamos, e incluso inconscientes, debido a estereotipos sobre diferencias sustanciales en las expectativas y el comportamiento frente al trabajo de hombres y mujeres que se refleja en las promociones y los salarios.

Otra de las razones está enfocada en que los hombres ocupan las posiciones de toma de decisiones en la mayoría de campos científicos. Pero el sesgo está ahí, incluso en comités de contratación con presencia femenina, donde se analizan carpetas con las mismas certificaciones y los candidatos hombres son los que obtienen mejores calificaciones e incluso mejores salarios. $\mathrm{Se}$ demuestra que el sesgo es generalizado (Moss-Racusin, Dovidio, Brescoll, Graham, \& Handelsan, 2012). Otra evidencia: Ya en el transcurso de la carrera, los hombres tienen un mayor reconocimiento público debido a su presencia en charlas y premios, y eso se puede ver reflejado en un salario más alto o mayor cantidad de fondos para investigación (Nittrour, y otros, 2018). Los hombres son mejores para los procesos de negociación en relación a su salario en comparación a las mujeres y tienen mayor acceso a becas frente a las mujeres $(28 \%)$, al menos en el Ecuador (Herdoiza, 2015).

En el Ecuador esta brecha existe, y conocemos que las tasas de subempleo y desempleo impactan principalmente a las mujeres. Sin tomar en cuenta que las mujeres trabajan 17 horas más a la semana en trabajo no remunerado (actividades asociadas a los quehaceres domésticos y de cuidado) (Herdoíza, 2015).

Eliminar las brechas de pago es fundamental para promover el avance equitativo de mujeres en las carreras asociadas a ciencia y tecnología. Por lo que es importante clarificar y transparentar las prácticas de compensaciones. La inclusión de mujeres en diferentes niveles de decisión y trabajo en equipo ha demostrado mayor nivel de apoyo y menos estereotipos (Nielsen, y otros, 2017). En cada una de las instituciones de educación superior, se debe asegurar las condiciones de igualdad del personal docente y administrativo, en relación a escalafones, remuneraciones y 
estabilidad. Tomar en cuenta los reglamentos, estatutos, así como la implementación de medidas de acción afirmativa (Herdoíza, 2015). Al momento de la contratación, tener un comité diverso, y si es posible con una revisión de pares ciegos al menos en los pasos iniciales del proceso.

Ya dentro de la institución, incluir políticas internas con temas relacionados con maternidad, aporte institucional para investigación, apoyo para padres jóvenes, acceso a guarderías y salas de lactancias (Reese, Harris-Tryin, Gill, \& Banaszynski, 2021). Apoyar el liderazgo femenino en diferentes ámbitos de la jerarquía institucional, que aportará también a la reducción de brechas de género. Realizar estudios sobre las brechas en las Universidades y replicar buenas prácticas (Reese, Harris-Tryin, Gill, \& Banaszynski, 2021).

\section{Apoyo a la mujer en tiempos de pandemia}

Ahora más que nunca debemos apoyar a las mujeres. La pandemia ha desafiado todas las estructuras de nuestra sociedad, pero los datos demuestran que las mujeres han sido especialmente afectadas por esta emergencia sanitaria y su impacto recién se verá en los años venideros. Muchas mujeres en ciencias están luchando por mantenerse a flote mientras cuidan a su familia y a sus hijos. Las mujeres en ciencias dedican más tiempo a los quehaceres domésticos que sus colegas, lo que reduce su tiempo de trabajo y eso generará un impacto a mediano y largo plazo en sus carreras. De ahí que nosotros debamos duplicar los esfuerzos para hacer una academia más inclusiva y amigable para las mujeres.
Los temas enfocados en equidad no se deben tratar de manera aislada, conllevan análisis más profundos relacionados con la edad, la educación, la cultura, el estrato socioeconómico, la etnia y la ubicación geográfica, entre otras. Generar espacios donde se hablen de estos temas, activar medidas y trabajar en conjunto para lograr la equidad en la ciencia y tecnología es un propósito posible de realizar en la academia y en toda la sociedad en su conjunto, porque ningún país está en la capacidad de negarse la posibilidad de la contribución intelectual del 50 \% de su población.

\section{LITERATURA CITADA}

Altamirano, G. (2020). Violencia de género en estudiantes universitarios: una mirada desde la determinación social. Quito: Maestría en Epidemiología y Salud.

Dasgupta, N., \& Stout, J. (2014). Girls and Women in Science, Technology, Engineering, and Mathematics: STEMing the Tide and Broadening Participation in STEM Careers. Behavioral and Brain Sciences, 1, 21-29

De Kleijn, M., Jayabalasingham, B., Falk-Krzesinski, H., Collins, T., Kuiper-Hoyng, L., Cingolani, I., . . . Roberge, G. (2020). The Researcher Journey Through a Gender Lens. An examination of research participation, career progression and perception across the globe. Elsevier.

Dimitriadi, A. (2013). Young women in science and technology: the importance of choice. Journal of Innovation and Entrepreneurship, 2(5), $1-14$. 
Dutt, K., Pfaff, D., Bernstein, A., Dillard, J., \& Block, C. J. (2016). Gender differences in recommendation letters for postdoctoral fellowships in geosciences. Nature Geosciences, 9, 805-808.

Else, H. (2018). Does science have a bullying problem? Nature, 563, 616-618.

Espinosa, K. (8 de Marzo de 2019). Revista Gestión. Obtenido de Revista Gestión: https://www.revistagestion.ec/sociedad-analisis/solo35-de-mujeres-en-ecuador-ocupa-cargos-directivos

Guerrero, S. (31 de marzo de 2021). Bullying académico: profesores e investigadores también lo sufren. Obtenido de Primicias: https://www. primicias.ec/noticias/firmas/bullying-academicoprofesores-investigadores-tambien-sufren-ecuador/? utm _ source =twitter\&utm_medium $=\& u t m_{-} \dagger$ erm $=\& u t m$ _ content $=\& u t m \_$campaign $=$

Herdoíza, M. (2015). Construyendo igualdad en la Educación Superior. Fundamentación y lineamientos para transversalizar los ejes de igualdad y ambiente (1 ed.). Quito: Senescyt/Unesco.

King, M., Bergstrom, C., Correll, S., Jacquet, J., \& West, J. (2017). Men set their own cites high: gender and self- citation across fields and over time. Socius: Sociological Research for a Dynamic World, 3, 1-22.

Lariviere, V., Chaoquin, N., Yves Gingras, B. C., \& Sugimoto, C. (2013). 2013. Global gender disparities in Science, 504 (7479), $211-213$.

Nielsen, M., Alegría, S., Borienson, L., Ełzkowithz, H., Flak-Krzesinski, H., Joshi, A., ... Schiebinger, L. (2017). Gender diversity leads to better science. Proceedings of the National Academy of Sciences, $114(8)$, 1740-1742.
Nittrour, C., Hebl, M., Ashburn-Nardo, L., Trump-Steele, R., Lane, D., \& Valian, V. (2018). Gender disparities in colloquium speakers at top universities. Proceedings of the National Academy of Science, 115(1), 104-108.

Nosek, B., Banaij, M., \& Greenwald, A. (2002). Math=male, $m e=$ female, therefore math not=me. Journal of Personality and Social Psychology, 83(1), 44-59.

Makarova, E., Aeschlimann, B., \& Herzog, W. (2019). The Gender Gap in STEM Fields: The Impact of the Gender Stereotype of Math and Science on Secondary Students' Career Aspirations. Frontiers in Education, 4(60).

Moss-Racusin, C., Dovidio, J., Brescoll, V., Graham, M., \& Handelsan J. (2012). Science faculty`s subtle gender biases favor male students. Proceedings of the National Academy of Science, 109(4), 16474-16479

OECD. (2015). Frascati Manual 2015: Guidelines for Collecting and Reporting Data on research and Experimental development, The Measurement of Scientific, Technological and Innovation Activities. Paris: OECD Publishing.

Organización de Estados Iberoamericanos. Observatorio CTS. (2020). El Estado de la Ciencia y Tecnología. Principales indicadores de Ciencia y Tecnología Iberoamericanos/Interamericanos 2020. OEl.

Ormaza, A. (2013). Prevalencia del Acoso Sexual en los estudiantes de la Escuela Superior de Chimborazo. Propuesta de intervención. Riobamba: Escuela Politécnica de Chimborazo.

Reese, T., Harris-Tryin, T. A., Gill, J., \& Banaszynski, L. (2021). Supporting women in academia during and after a global pandemic. Science Advances, 7. 
Secretaria Técnica Planifica Ecuador. (2019). Informe de avance del Cumplimiento de la Agenda 2030 para el desarrollo sostenible. Quito: Secretaría Técnica Planifica Ecuador.

Somerville, L., \& Gruber, J. (16 de October de 2020). Three trouble spots facing women in science- and how we can tackle them. Science.

Unesco. (2015). UNESCO SCIENCE REPORT, Towards 2020. París: UNESCO.

Unesco Institute for Statistics. (2019). Women in Science. Unesco .

Weisgram, E. (2019). Reducing Gender Stereotypes in Toys and Play for Smarter, Stronger and Kinder Kids. American Journal of Play, 12(1), 74-88.

Zambrano Mendoza, J. (2020). La Sociedad del Conocimiento: Cantidad, Categoría y Género de los Investigadores del Ecuador. Mundos Plurales -Revista Latinoamericana de Política y Acción Pública, 6(1), 73-92.

\section{BIOGRAFÍA DE LA AUTORA}

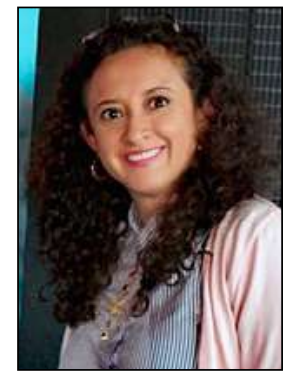

CLAUDIA SEGOVIA

Obtuvo su Licenciatura en Biología en la Pontificia Universidad Católica del Ecuador. Su maestría la realizó en Ohio University en Ambiente y Biología Vegetal y su PhD en el Departamento de Botánica de University of Florida. Su investigación se centra en los ecosistemas altoandinos con énfasis en su evolución y conservación. Durante su carrera se ha vinculado a diferentes grupos de Mujeres en Ciencia, fue miembro activo de WISE-UF durante sus estudios de posgrado. 